\title{
ROTATION STATE OF COMET 103P/HARTLEY 2 FROM RADIO SPECTROSCOPY AT 1 mm*
}

\author{
Michae Drahus $^{1}$, David JewitT ${ }^{1}$, Aurélie Guilbert-Lepoutre ${ }^{1}$, Waceaw Waniak ${ }^{2}$, James Hoge ${ }^{3}$, Dariusz C. Lis ${ }^{4}$, \\ Hiroshige Yoshida ${ }^{5}$, Ruisheng Peng ${ }^{5}$, and Albrecht Sievers ${ }^{6}$ \\ ${ }^{1}$ Department of Earth and Space Sciences, University of California at Los Angeles, Los Angeles, CA 90095, USA; mdrahus@ucla.edu \\ ${ }^{2}$ Astronomical Observatory, Jagiellonian University, Kraków, Poland \\ ${ }^{3}$ Joint Astronomy Centre, Hilo, HI 96720 , USA \\ ${ }^{4}$ Division of Physics, Mathematics, and Astronomy, California Institute of Technology, Pasadena, CA 91125, USA \\ ${ }^{5}$ Caltech Submillimeter Observatory, Hilo, HI 96720, USA \\ ${ }^{6}$ Instituto de Radio Astronomìa Milimétrica, Granada, Spain \\ Received 2011 March 7; accepted 2011 March 30; published 2011 May 16
}

\begin{abstract}
The nuclei of active comets emit molecules anisotropically from discrete vents. As the nucleus rotates, we expect to observe periodic variability in the molecular emission line profiles, which can be studied through millimeter/ submillimeter spectroscopy. Using this technique we investigated the $\mathrm{HCN}$ atmosphere of comet 103P/Hartley 2, the target of NASA's EPOXI mission, which had an exceptionally favorable apparition in late 2010. We detected short-term evolution of the spectral line profile, which was stimulated by the nucleus rotation, and which provides evidence for rapid deceleration and excitation of the rotation state. The measured rate of change in the rotation period is $+1.00 \pm 0.15$ minutes day ${ }^{-1}$ and the period itself is $18.32 \pm 0.03 \mathrm{hr}$, both applicable at the epoch of the $E P O X I$ encounter. Surprisingly, the spin-down efficiency is lower by two orders of magnitude than the measurement in comet 9P/Tempel 1 and the best theoretical prediction. This secures rotational stability of the comet's nucleus during the next few returns, although we anticipate a catastrophic disruption from spin-up as its ultimate fate.
\end{abstract}

Key words: comets: general - comets: individual (103P/Hartley 2) - radio lines: general

\section{INTRODUCTION}

Comet 103P/Hartley 2 (hereafter 103P) is a Jupiter-family comet (JFC), which currently has a $6.47 \mathrm{yr}$ orbital period and perihelion at $1.06 \mathrm{AU}$. On UT 2010 October 20.7 it reached the minimum geocentric distance of only $0.12 \mathrm{AU}$, making by far the closest approach to the Earth since its discovery (Hartley 1986), and becoming a naked-eye object. Shortly after, on UT 2010 November 4.6, the comet was visited by NASA's EPOXI spacecraft, which approached the nucleus within $700 \mathrm{~km}$ and provided detailed images and spectra (A'Hearn et al. 2011). Both the ground-based data, obtained at the unusually favorable geometry, and the unique observations carried out by the spacecraft create an exceptional platform for new groundbreaking investigations.

Taking advantage of this unique opportunity we addressed one of the burning problems of planetary science, which is the characteristic lifetime of active cometary nuclei. They can be dynamically ejected out of the sublimation zone, or decay through collision, tidal disruption, devolatilization, deactivation, disintegration, and - the least understood - spontaneous fragmentation (Jewitt 1992). Of several scenarios proposed to explain the last process, a notable one is rotational breakup, which must occur for spinning bodies when the centripetal force surpasses self-gravity and material strength (e.g., Davidsson 2001). Interestingly, models show that torques exerted by gas jets can significantly increase or decrease the rotation rate during a single orbit (e.g., Gutiérrez et al. 2002), although the magnitude strongly depends on the nucleus size and activity, and the poorly understood effective moment arm. The first unambiguous measurement of this effect was obtained only recently for comet 9P/

\footnotetext{
* Based on observations carried out with the IRAM $30 \mathrm{~m}$, JCMT $15 \mathrm{~m}$, and CSO $10.4 \mathrm{~m}$ telescopes. IRAM is supported by INSU/CNRS (France), MPG (Germany), and IGN (Spain). JCMT is operated by Joint Astronomy Centre and supported by STFC (UK), NRC (Canada), and NWO (Netherlands). CSO is operated by Caltech and supported through NSF grant AST-0540882 (USA).
}

Tempel 1, which was found to be slowly spinning up (Belton \& Drahus 2007; Belton et al. 2011). Clearly, further observational studies of the dynamics of cometary rotation are essential to establish the nucleus lifetime with respect to rotational breakup.

Since the influence of jets must be strongest for small, elongated, and active comets (e.g., Samarasinha et al. 1986; Jewitt 1999; Drahus \& Waniak 2006), the nucleus of 103P is an excellent test body: it is one of the smallest known JFCs (equivalent radius $\sim 600 \mathrm{~m}$ ), it is elongated and active (Lisse et al. 2009; A'Hearn et al. 2011), and hence it was predicted to show measurable period changes during the last return (Drahus 2009; Lisse et al. 2009). In general, however, it is very difficult to investigate the rotation of an active nucleus; especially of a very small one and from the ground-securing simultaneously the necessary measurement precision. Our earlier studies show that perhaps the best possibilities are offered by millimeter/submillimeter spectroscopy (Drahus et al. 2010). When the molecules are emitted from discrete vents, or the body is irregular, the diurnal rotation modulates the emission line profiles, which can be conveniently studied in a velocity-resolved spectral time series. The effect will be best visible when every single spectrum results from the molecules released at similar nucleus rotation phases, which is satisfied when the molecules leave the projected telescope beam on a timescale much shorter than the body rotation period. The best candidates are hence nearby comets rotating slowly, and observed ideally in small beams using short integration times with the largest antennas. Consequently, we identified $103 \mathrm{P}$ as a terrific target for large ground-based millimeter/ submillimeter telescopes (Drahus 2009), owing to the unusually small geocentric distance, localized activity, and more than sufficiently slow rotation (the last two confirmed after the fact).

\section{OBSERVATIONS}

Between early September and mid 2010 December we used all large single-dish ground-based millimeter/submillimeter 


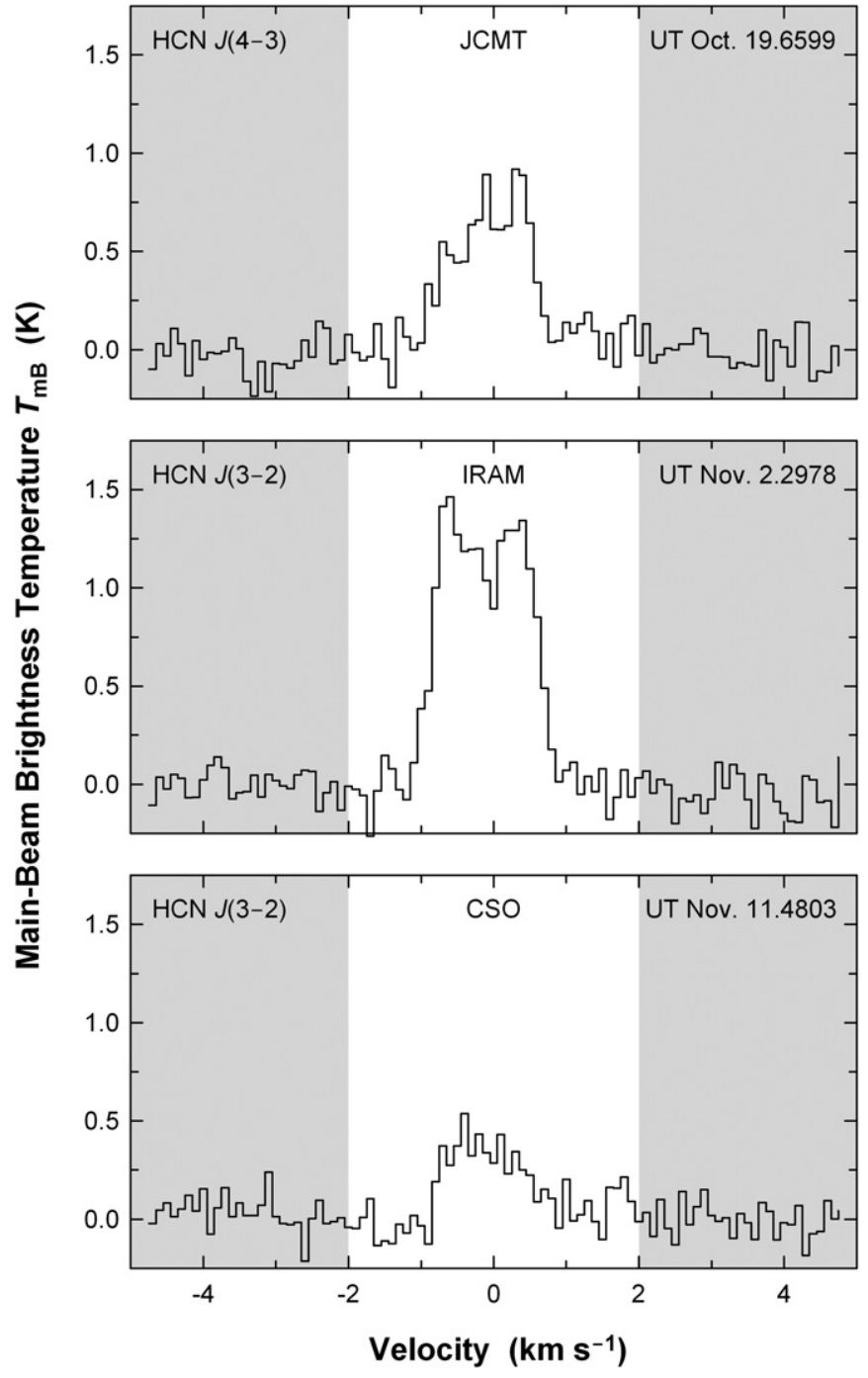

Figure 1. Example of HR spectra. The unshaded region was used for calculations.

facilities operating around one millimeter and offering open time, to carry out spectral monitoring of $\mathrm{HCN}$. This molecule is a particularly good tracer of the cometary rotation (Drahus et al. 2010) thanks to its well-established origin directly from the nucleus and because it has by far the brightest emission lines in the one-millimeter atmospheric windows. Our campaign was the first at these wavelengths specifically designed to investigate the rotation state of a comet, and thus provided an unprecedentedly rich and dense velocity-resolved spectral time series of a cometary parent molecule.

In this work, we use 438 spectra of the $J(3-2)$ and $J(4-3)$ rotational transitions (Figure 1), collected with IRAM, JCMT, and CSO on 20 nights between UT 2010 September 29.3 and December 15.6 (Table 1). The spectra were calibrated in the standard manner; each covers 10-15 minutes, and was analyzed at spectral resolutions of $0.10 \mathrm{~km} \mathrm{~s}^{-1}$ (high resolution; HR) and $0.25 \mathrm{~km} \mathrm{~s}^{-1}$ (low resolution; LR). Details of the observations and reductions will be presented in a subsequent paper.

\section{ANALYSIS}

The HR spectra were first parameterized in terms of the line area and center velocity and then converted to physical quantities: $\mathrm{HCN}$ production rate $Q$ and median radial $\mathrm{HCN}$ gas-
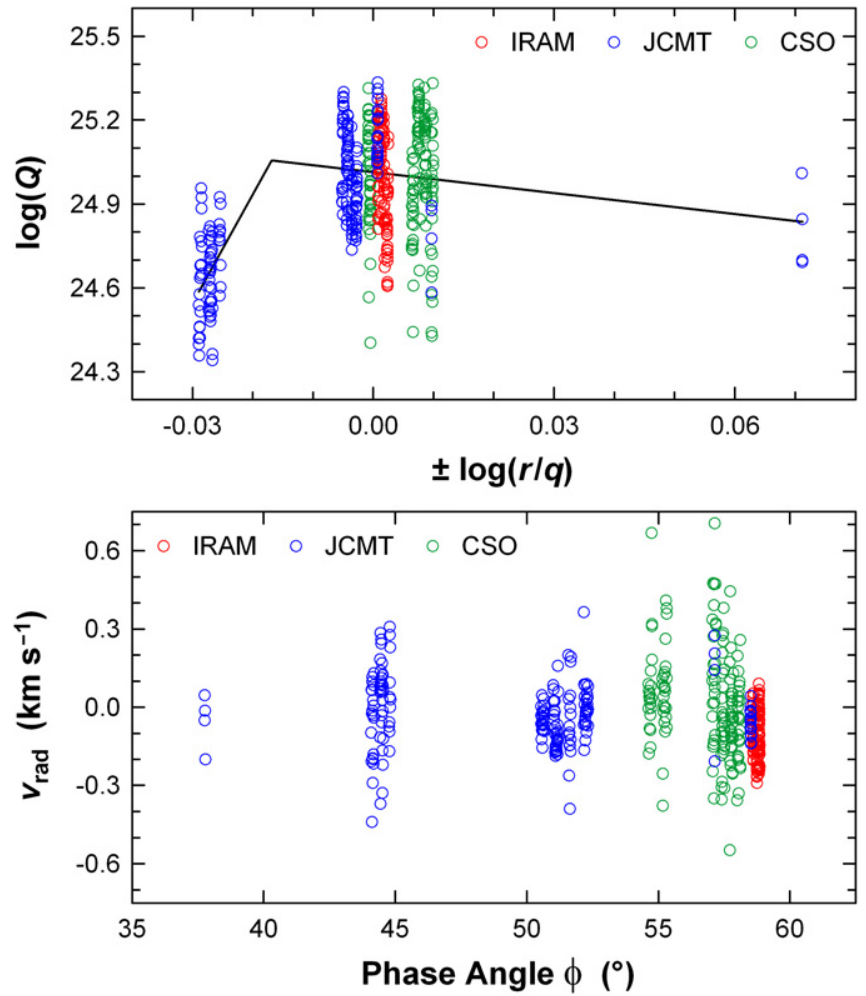

Figure 2. Orbital trends in the data. Top panel: dependence of the production rate $Q$ on heliocentric distance $r$ (normalized to the perihelion distance $q$ ) in a $\log -\log$ space. Negative $\log (r / q)$ indicate positions before perihelion. The solid line shows a semi-empirical fit that was used to remove the heliocentric trend and calculate the production-rate deviations $\Delta \log (Q)$. Bottom panel: behavior of the median radial velocity $v_{\text {rad }}$ with phase angle $\phi$. Note: the EPOXI encounter occurred at $\log (r / q)=0.002$ and $\phi=58.8$.

flow velocity $v_{\text {rad }}$. All these steps were performed following our standard methods and procedures (Drahus 2009; Drahus et al. 2010), and will be described in a subsequent paper. In this work, we only highlight our approach to estimate errors (Appendix A).

Although unavoidably affected by model simplifications, the physical quantities are naturally free (to the first order) of trivial instrumental, topocentric, and transition-specific differences, hence making the data set fairly homogeneous and suitable for the investigation of variability. Figure 2 shows the evolution of $Q$ with heliocentric distance $r$, and displays $v_{\text {rad }}$ against phase angle $\phi$.

The first trend in Figure 2 was measured for several comets before (e.g., Biver et al. 2002a; Drahus et al. 2010). We see an increase in the production rate as 103P approaches perihelion and a decrease afterward, with superimposed short-term variability responsible for the excessive scatter. The maximum mean-diurnal level of $\mathrm{HCN}$ is about $1 \times 10^{25} \mathrm{molec} \mathrm{s}^{-1}$, which seems high for a JFC but not abnormal for a comet (Figure 3). The ratio of $\mathrm{HCN}$ to $\mathrm{H}_{2} \mathrm{O}$ (Biver et al. 2010; A'Hearn et al. 2011) at the level of $0.1 \%$ is also typical of comets (Biver et al. 2002b; Bockelée-Morvan et al. 2004). After subtracting the heliocentric trend, we derived production-rate deviations $\Delta \log (Q)$ about the mean-diurnal level.

The expected correlation of $v_{\text {rad }}$ with $\phi$ has been observationally confirmed only recently (Drahus et al. 2010); however, in our data we do not find any trend (Figure 2) because of the highly limited phase-angle coverage, short-term variations, and observing noise. 
Table 1

Journal of Observations

\begin{tabular}{|c|c|c|c|c|c|c|c|c|}
\hline $\begin{array}{l}\text { UT Date } 2010 \\
\text { (mid time) }\end{array}$ & Telescope & $\begin{array}{c}\text { Coverage }^{\mathrm{a}} \\
(\mathrm{hr})\end{array}$ & Num. ${ }^{b}$ & $\begin{array}{c}r^{\mathrm{c}} \\
(\mathrm{AU})\end{array}$ & $\begin{array}{c}\Delta^{\mathrm{d}} \\
(\mathrm{AU})\end{array}$ & $\begin{array}{l}\phi^{\mathrm{e}} \\
\left({ }^{\circ}\right)\end{array}$ & $\begin{array}{c}\text { Beam }^{\mathrm{f}} \\
(\mathrm{km})\end{array}$ & $\begin{array}{c}\tau_{\perp} \mathrm{g} \\
\text { (minutes) }\end{array}$ \\
\hline Sep 29.4795 & JCMT & 6.6 & 20 & 1.1310 & 0.1926 & 44.1 & 958 & 20.0 \\
\hline Sep 30.4229 & JCMT & 8.1 & 28 & 1.1265 & 0.1873 & 44.5 & 932 & 19.4 \\
\hline Oct 1.2865 & JCMT & 2.8 & 11 & 1.1225 & 0.1826 & 44.8 & 908 & 18.9 \\
\hline Oct 16.6228 & JCMT & 6.5 & 21 & 1.0710 & 0.1241 & 50.6 & 617 & 12.9 \\
\hline Oct 17.5706 & JCMT & 10.0 & 33 & 1.0691 & 0.1228 & 51.1 & 611 & 12.7 \\
\hline Oct 18.5236 & JCMT & 5.0 & 16 & 1.0673 & 0.1218 & 51.6 & 606 & 12.6 \\
\hline Oct 19.5997 & JCMT & 8.3 & 27 & 1.0655 & 0.1211 & 52.3 & 602 & 12.5 \\
\hline Oct 23.6593 & $\mathrm{CSO}$ & 6.7 & 20 & 1.0606 & 0.1225 & 54.7 & 1249 & 26.0 \\
\hline Oct 24.5773 & $\mathrm{CSO}$ & 8.3 & 24 & 1.0599 & 0.1236 & 55.2 & 1260 & 26.3 \\
\hline Nov 1.6117 & JCMT & 6.1 & 20 & 1.0604 & 0.1447 & 58.5 & 720 & 15.0 \\
\hline Nov 2.2058 & IRAM & 7.9 & 24 & 1.0609 & 0.1469 & 58.6 & 470 & 9.8 \\
\hline Nov 3.1957 & IRAM & 8.5 & 26 & 1.0619 & 0.1506 & 58.7 & 482 & 10.1 \\
\hline Nov 4.1974 & IRAM & 8.4 & 25 & 1.0631 & 0.1546 & 58.8 & 495 & 10.3 \\
\hline Nov 5.1820 & IRAM & 8.7 & 28 & 1.0644 & 0.1586 & 58.8 & 508 & 10.6 \\
\hline Nov 10.6112 & $\mathrm{CSO}$ & 7.8 & 24 & 1.0748 & 0.1829 & 58.1 & 1864 & 38.8 \\
\hline Nov 11.5986 & $\mathrm{CSO}$ & 8.2 & 30 & 1.0773 & 0.1876 & 57.8 & 1912 & 39.8 \\
\hline Nov 12.6163 & $\mathrm{CSO}$ & 7.8 & 28 & 1.0800 & 0.1924 & 57.5 & 1962 & 40.9 \\
\hline Nov 13.5404 & JCMT & 0.8 & 4 & 1.0826 & 0.1969 & 57.1 & 980 & 20.4 \\
\hline Nov 13.5903 & $\mathrm{CSO}$ & 7.4 & 25 & 1.0827 & 0.1972 & 57.1 & 2010 & 41.9 \\
\hline Dec 15.6115 & JCMT & 0.8 & 4 & 1.2471 & 0.3650 & 37.8 & 1816 & 37.8 \\
\hline
\end{tabular}

Notes. IRAM and CSO observed HCN J(3-2) and JCMT observed HCN J(4-3).

a Time Span between the mid moments of the first and last spectrum.

b Number of collected spectra.

c Heliocentric distance.

d Topocentric distance.

e Topocentric phase angle.

${ }^{\mathrm{f}}$ HWHM of the main beam at comet distance; at the observed frequencies the HWHM is 4". 4 for IRAM, 6.'9 for JCMT, and 14". 1 for CSO.

g Minimum escape time from the main beam, needed to reach HWHM with the assumed constant velocity of $0.8 \mathrm{~km} \mathrm{~s}{ }^{-1}$.

\section{NUCLEUS ROTATION}

While the short-term variations could be sporadic (A'Hearn et al. 2005), it is also entirely possible that they were stimulated by the nucleus rotation. We pursued this issue using an innovative technique: the Dynamized Structural Periodicity Analysis (DSPA), which we created specifically for this purpose (Appendix B). The method was developed from our Dynamized Periodicity Analysis (DPA), which-utilizing classical algorithms for a time series of signals-introduces a dynamical formula for the body rotation and rigorous weighting of the input data (Drahus \& Waniak 2006). DPA determines the rotation frequency simultaneously with its time derivative from the entire data set, taking advantage of the maximum available time base, and therefore is far superior compared to separate classical (constant) period determinations from smaller subsets. Recently DPA demonstrated excellent sensitivity in providing important evidence for the slow spin-up of comet 9P/Tempel 1 (Belton \& Drahus 2007; Belton et al. 2011). DSPA goes further, and analyzes periodicity not only in the level but in the complete structure of the signal in the input data, properly combining the information from independent data channels. At the same time it keeps all the properties of DPA, and for a single-channel time series fully reduces to the latter. The technique does not rely on any variability models, neither versus time nor across the channels. Although DSPA allows for any a priori law controlling the effective torque, in this analysis we assumed it was constant.

Using the derived production-rate variations $\Delta \log (Q)$, we normalized the original spectra to eliminate all the trivial effects and remove the physical heliocentric trend (Figure 2). In order to secure an affordable computation time and reasonable signal-tonoise ratio in the individual data channels, DSPA was applied

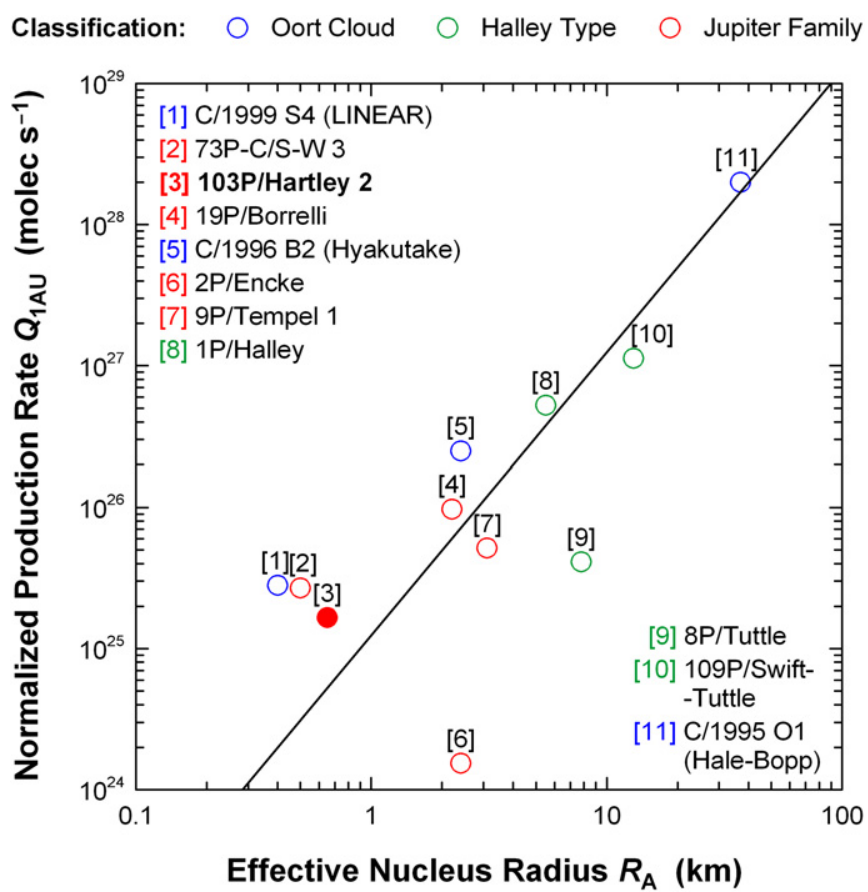

Figure 3. Compilation of the normalized $\mathrm{HCN}$ production rates $Q_{1 \mathrm{AU}}$ for comets with known area-equivalent nucleus radius $R_{\mathrm{A}}$, presented in a $\log -\log$ space (see Drahus et al. 2010, and references therein). The production rates were normalized to the common heliocentric distance $r=1$ AU using the canonical $Q(r) \sim r^{-4}$ whenever needed. The solid line is a square function fitted in a $\log -\log$ space. It represents a typical (constant) active fraction of the nucleus.

to the normalized LR spectra. We used 17 channels from the velocity interval between -2 and $+2 \mathrm{~km} \mathrm{~s}^{-1}$, which is more 


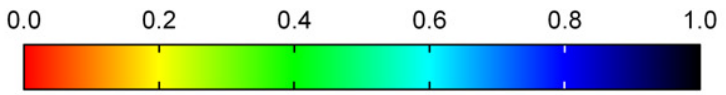

Solutions: $\quad+$ PDM $\times$ HF $\bigcirc$ Mean 1-Peak $\bigcirc$ Expected 3-Peak
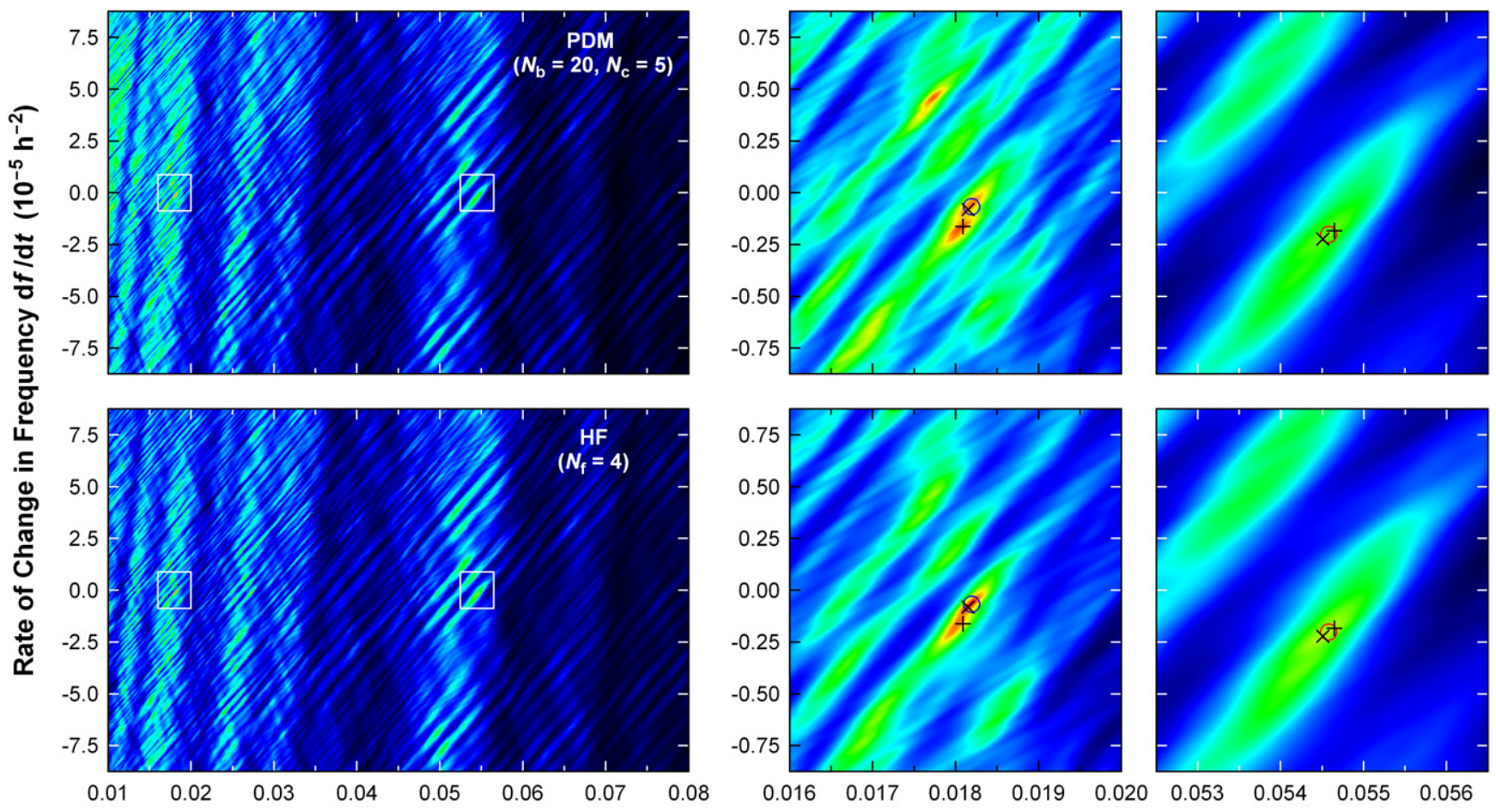

Frequency of Periodicity at the EPOXI Encounter $f_{0}\left(\mathrm{~h}^{-1}\right)$

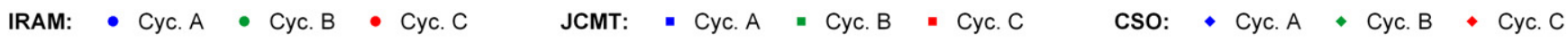
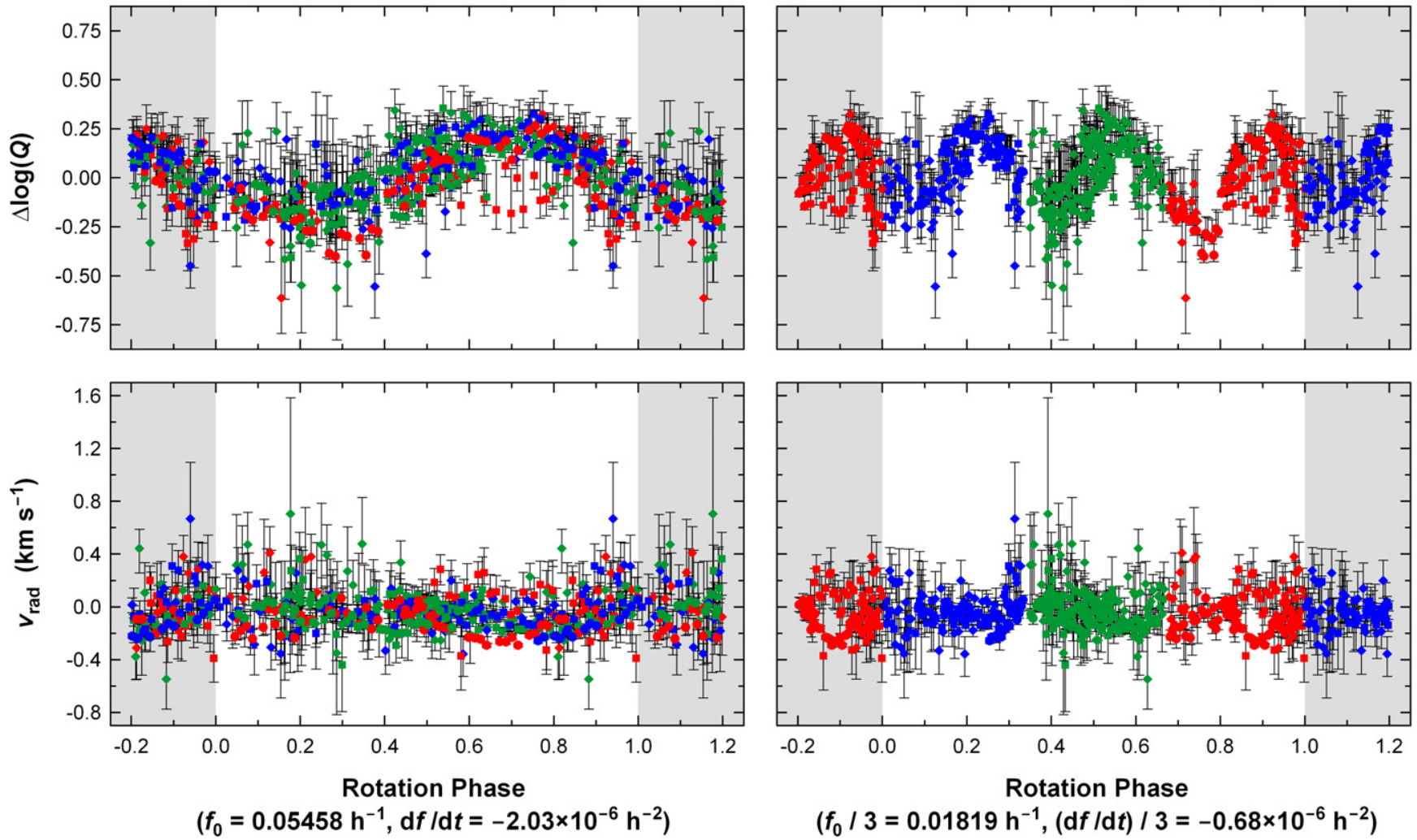

Figure 4. Results of DSPA. Upper panels: dynamical periodograms for the regions of investigated space. Linear color scale denotes $\theta_{\text {disp }}=\log \left(\eta\left(\bar{\theta}-\bar{\theta}_{\text {min }}\right) /\left(\bar{\theta}_{\text {max }}-\right.\right.$ $\left.\left.\bar{\theta}_{\min }\right)+10-\eta\right)$, where $\eta=9$ and function $\bar{\theta}=\bar{\theta}(f, d f / d t)$ is defined in Appendix B. Lower panels: rotation phase profiles for $\Delta \log (Q)$ and $v_{\text {rad }}$ for two periodicity solutions (see the text). The shaded regions indicate duplications of the phase space. The EPOXI encounter occurred during Cycle B at the rotation phase of 0.5 . 
than enough to adequately sample the line profile. They were analyzed with two well-established kernels (Drahus \& Waniak 2006): weighted version of Phase Dispersion Minimization (PDM) and weighted Harmonic Fit (HF).

Figure 4 presents dynamical periodograms resulting from the analysis. The best single-peak solution is found for the rotation frequency $f_{0}=0.0546 \pm 0.0001 \mathrm{hr}^{-1}$ (given at the epoch of the EPOXI encounter; indicated by index zero) and frequency time derivative $d f / d t=(-2.0 \pm 0.3) \times 10^{-6} \mathrm{hr}^{-2}$. It is equivalent to the period of $18.32 \pm 0.03 \mathrm{hr}$ increasing at the relative rate of $0.07 \% \pm 0.01 \%$ (e.g., $1.00 \pm 0.15$ minutes day $^{-1}$, or $0.75 \pm$ 0.10 minutes cycle ${ }^{-1}$ ), both applicable at the EPOXI encounter. The result is insensitive to the uncertainties resulting from the earlier reductions-including the removal of the heliocentric trend, as the data most affected enter the analysis with very low statistical weights.

We note that the global solution is very close to the third multiple of the above single-peak solution, which means that the pattern of $\mathrm{HCN}$ production repeats significantly better every three rotation cycles. We explain this behavior by excitation of the rotation state: every three cycles of the fundamental periodicity the independent rotation modes coincide at similar phases and then the three-cycle repeats. But the resonance may not be perfect, as the spectra from the same rotation phases and corresponding cycles still show differences in the line profiles. (For example, Figure 1 shows spectra obtained during Cycle B at the rotation phase 0.5 ( $\mathrm{cf}$. Figure 4 ), corresponding to the moment of the EPOXI encounter.) This effect could be caused by small differences in the instantaneous nucleus orientation (implying differences in the direction of gas flow), although alternative explanations may be possible as well. Interestingly, the larger nucleus of comet 9P/Tempel 1 was found close to a fully relaxed rotation state (Belton et al. 2011), while the even bigger nucleus of $1 \mathrm{P} /$ Halley was in a significantly excited state (Belton et al. 1991).

Figure 4 also shows the production-rate variations $\Delta \log (Q)$ and median radial gas-flow velocities $v_{\text {rad }}$ phased according to the best single-peak solution and its third multiple. The line variability comes primarily from the diurnal changes in the production rate, which follow a nearly sinusoidal trend. The single-peak solution is consistent with a single strong active area being the primary source of HCN. As the EPOXI encounter occurred at the rotation phase of 0.5 , when the $\mathrm{HCN}$ production rate was shortly before the diurnal maximum, we suppose that this area coincides with the strongest jet observed by the spacecraft (A'Hearn et al. 2011). This also implies that the higher-order multi-peak solutions, including the triple-peak third multiple indicated by the global minimum, cannot be plausibly associated with the fundamental periodicity.

\section{DISCUSSION AND CONCLUSIONS}

The obtained solution for periodicity indicates a rapid spindown of 103P's nucleus. Spin-down at a comparable rate was suggested earlier for comet C/2001 K5 (Drahus \& Waniak 2006), but the robust measurement obtained for 9P/Tempel 1 indicates spin-up at a 20 times smaller rate (Belton \& Drahus 2007; Belton et al. 2011). Dynamics of the rotation rate can be interpreted in terms of a simple model (e.g., Samarasinha et al. 1986; Jewitt 1999; Drahus \& Waniak 2006), where the rate of change in frequency $d f / d t$ is given by

$$
d f / d t=\frac{15}{16 \pi^{2}} \frac{v_{\mathrm{s}} Q_{\mathrm{tot}}}{R_{\mathrm{V}}^{4} \rho} \kappa,
$$

where $v_{\mathrm{s}}$ is the gas sublimation velocity, $Q_{\text {tot }}$ is the (meandiurnal) production rate of all gaseous species, $R_{\mathrm{V}}$ is the volumeequivalent nucleus radius, $\rho$ is the nucleus bulk density, and $\kappa$ is the dimensionless effective moment arm which is a measure of acceleration efficiency; $\kappa$ is negative for spin-down and positive for spin-up, and may exceed unity for non-spherical bodies (Drahus \& Waniak 2006). Substituting the measured $d f / d t=$ $-2.0 \times 10^{-6} \mathrm{hr}^{-2}$, and $Q_{\text {tot }}=Q_{\mathrm{H}_{2} \mathrm{O}}+Q_{\mathrm{CO}_{2}}=450 \mathrm{~kg} \mathrm{~s}^{-1}$ (A'Hearn et al. 2011), $v_{\mathrm{s}}=400 \mathrm{~m} \mathrm{~s}^{-1}$ (Combi et al. 2004), $\rho=400 \mathrm{~kg} \mathrm{~m}^{-3}$ (Richardson et al. 2007), and $R_{\mathrm{V}}=580 \mathrm{~m}$ (Lisse et al. 2009; A'Hearn et al. 2011), we find $\kappa=-0.04 \%$. The same equation yields $\kappa=+4 \%$ for the properties of comet 9P/Tempel $1\left(R_{\mathrm{V}}=3000 \mathrm{~m}\right.$ (A'Hearn et al. 2005), $Q_{\text {tot }}=$ $Q_{\mathrm{H}_{2} \mathrm{O}}+Q_{\mathrm{CO}_{2}}=160 \mathrm{~kg} \mathrm{~s}^{-1}$ (Feaga et al. 2007), and $d f / d t=$ $+9.7 \times 10^{-8} \mathrm{hr}^{-2}$ (Belton et al. 2011); otherwise the same as above), which is consistent with a model prediction $|\kappa| \sim 5 \%$ (Jewitt 1999). Even though $\kappa$ in 103P is two orders of magnitude lower compared to $9 \mathrm{P}$, the comet changes its rotation frequency a factor of 20 faster. This is understandable from Equation (1), since $d f / d t$ has a strong dependence on the nucleus size, and the nucleus of $103 \mathrm{P}$ is tiny compared to $9 \mathrm{P}$. Moreover, 103P is more active per unit surface area (Figure 3) and approaches the Sun closer, therefore it has a higher total sublimation rate at perihelion, in spite of the much smaller total area.

If the pattern of mass loss remains stable, the above result lets us crudely speculate about the rotational past and future of $103 \mathrm{P}$ on the current orbit. We estimate the orbit-integrated change of rotation frequency at the level of $0.012 \mathrm{hr}^{-1}$ and assume the critical rotation period of approximately $3 \mathrm{hr}$ (Davidsson 2001). This allows for no more than $\sim 20$ previous revolutions. At the present rate, the nucleus will stop rotating during the fourth or fifth return from now (perihelion in 2036 or 2043). If it survives the increased thermal stress, it should start spinning up in the opposite direction and reach the rotational disruption limit $\sim 25$ orbits later (year $\sim 2200$ ). We note that rotational breakup has by far the shortest timescale determining the lifetime of such a small object (Jewitt 1992, 1999; Lisse et al. 2009). Moreover, bearing in mind the location of the main active area on the nucleus (A'Hearn et al. 2011), even a small change in the jet configuration may dramatically increase the effective moment arm-bringing 103P to the end of its life within one or few orbits.

We expect that small, young, and naturally volatile-rich comets, having typical effective moment arms of the order of $5 \%$, routinely experience rotational instability and fragmentation right after being injected to the sublimation zone yet before being discovered. In this way the observed flat size distribution of comet nuclei (e.g., Snodgrass et al. 2011) can be naturally explained. With its tiny and active nucleus, 103P appears as a lucky survivor, protected for some limited time from rotational disruption by the remarkably low factor $\kappa$.

We thank M. Polińska for providing resources and the telescope staff for their excellent work. This project was supported by NASA through a Planetary Astronomy Program grant to D.J.

Facilities: IRAM:30m (EMIR), JCMT (HARP), CSO (ZRex).

\section{APPENDIX A}

\section{ERROR EVALUATION}

We have examined all error sources and concluded that the signal uncertainty in the individual spectral channels is dominated by observing noise and imperfect pointing. 
Observing noise was estimated from signal variations about the baseline assuming a normal distribution. Since noise is nearly entirely controlled by the sky and receiver temperatures (surpassing the comet antenna temperature by about two orders of magnitude), we assumed the same noise level inside the line window. Noise dependence on frequency is negligible in this extremely narrow spectral range.

The influence of pointing errors was estimated from a simple model, which we have developed for this purpose from our earlier constructions (Drahus et al. 2010). For each telescope axis we assume a normally distributed pointing error with a standard deviation of $2^{\prime \prime}$ for IRAM and JCMT, and $3^{\prime \prime}$ for CSO; it is easy to show that this is equal to the most probable total pointing offset. We also adopt a simple steady-state isotropic coma model with infinite molecular lifetime and brightness proportional to the number of molecules. Under these assumptions, we calculated probability density functions (PDFs) of signal loss for each telescope and neglected other (less significant) effects from imperfect pointing.

To calculate the errors of all the parameters of interest, we followed our Monte Carlo approach (Drahus \& Waniak 2006; Drahus et al. 2010) and generated 500 simulations using the derived PDFs. A starting point for each simulation was an original spectrum corrected for the most probable signal loss from pointing. To each spectral channel we added a random realization of noise and then scaled the complete spectrum according to a random realization of signal loss from pointing. Then, by analyzing the parameters' variations about their original values, we derived their standard errors.

The signal errors in the individual spectral channels feature several interesting properties. With signal approaching zero they asymptotically approach the noise limit, and oppositely, they approach the pointing limit with the signal increasing to infinity. The pointing component is proportional to signal whereas the noise component does not depend on signal. While the noise component can be controlled (reduced) by spectral or temporal binning, the pointing component cannot, because it affects all the channels in one spectrum in the same way, and similarly several spectra taken within short time (the latter not being taken into account by our simple model). For this reason the pointing component should not be included when analyzing signal variability across the channels. Moreover, the error from pointing is nearly one-sided (positive) while the one from noise is symmetric.

Because several assumptions of this model may not be well satisfied, the provided uncertainties should be considered as crude estimates. Nevertheless, to our best knowledge it is the first approach to quantify how the imperfect pointing of radio telescopes affects the observed spectra.

\section{APPENDIX B}

\section{DYNAMIZED STRUCTURAL PERIODICITY ANALYSIS}

The DSPA method is applied to a time series of $M$ measurements. Quality of data phasing is measured by a function $\theta$, which is a variance ratio of the phased and unphased data. DSPA calculates functions $\theta_{i}(f, d f / d t)$ of DPA (Drahus \& Waniak 2006) for every data channel $i$ and averages the results from the considered $N$ channels to provide $\bar{\theta}(f, d f / d t)$ :

$$
\bar{\theta}=\frac{\sum_{i=1}^{N} \theta_{i} W_{i}}{\sum_{i=1}^{N} W_{i}}
$$

using weights $W_{i}$ defined as square-mean signal over meansquare error:

$$
W_{i}=\frac{{\overline{S_{i}}}^{2}}{\overline{\sigma_{i}^{2}}}=\left(\frac{\sum_{j=1}^{M} S_{i j} w_{i j}}{\sum_{j=1}^{M} w_{i j}}\right)^{2} \frac{M}{\sum_{j=1}^{M} \sigma_{i j}^{2}},
$$

where $S_{i j}$ is the signal in $i$ th data channel and $j$ th time moment, $\sigma_{i j}$ is its standard error, and $w_{i j}=\sigma_{i j}^{-2}$. Note that both functions $\theta_{i}$ used by DPA, i.e., the weighted version of PDM and the weighted HF, use the same weights $w_{i j}$ as above. Since our signal errors are asymmetric, we used their mean-square values for this purpose.

Weighting with $W_{i}$ implemented in DSPA naturally prefers channels with strong signal, making the method insensitive, from the theoretical point of view, to the selection of line window (as long as the line is fully inside); channels having on average zero signal contribute to $\bar{\theta}$ with zero weights. Moreover, it prefers channels with small uncertainties, which is particularly important in this application since imperfect pointing differentiates signal error across the channels; for identical uncertainties in every channel this term cancels out. It is therefore clear that in our case the effective channel weight is established by the fine balance between channel signal and noise-as the error from pointing is proportional to the signal.

\section{REFERENCES}

A'Hearn, M. F., et al. 2005, Science, 310, 258

A'Hearn, M. F., et al. 2011, Science, in press

Belton, M. J., \& Drahus, M. 2007, AAS DPS, 39, 498

Belton, M. J. S., Mueller, B. E. A., Julian, W. H., \& Anderson, A. J. 1991, Icarus, 93, 183

Belton, M. J. S., et al. 2011, Icarus, 213, 345

Biver, N., Bockelée-Morvan, D., Crovisier, J., Lecacheux, A., Frisk, U., Floren, H.-G., \& Hjalmarson, A. 2010, CBET, 2524

Biver, N., et al. 2002a, Earth Moon Planets, 90, 5

Biver, N., et al. 2002b, Earth Moon Planets, 90, 323

Bockelée-Morvan, D., Crovisier, J., Mumma, M. J., \& Weaver, H. A. 2004, in Comets II, ed. M. C. Festou, H. U. Keller, \& H. A. Weaver (Tucson, AZ: Univ. Arizona Press), 391

Combi, M. R., Harris, W. M., \& Smyth, W. H. 2004, in Comets II, ed. M. C. Festou, H. U. Keller, \& H. A. Weaver (Tucson, AZ: Univ. Arizona Press), 523

Davidsson, B. J. R. 2001, Icarus, 149, 375

Drahus, M. 2009, PhD thesis, Univ. Göttingen

Drahus, M., Küppers, M., Jarchow, C., Paganini, L., Hartogh, P., \& Villanueva, G. L. 2010, A\&A, 510, A55

Drahus, M., \& Waniak, W. 2006, Icarus, 185, 544

Feaga, L. M., A'Hearn, M. F., Sunshine, J. M., Groussin, O., \& Farnham, T. L. 2007, Icarus, 191, 134

Gutiérrez, P. J., Ortiz, J. L., Rodrigo, R., López-Moreno, J. J., \& Jorda, L. 2002, Earth Moon Planets, 90, 239

Hartley, M. 1986, IAU Circ., 4197

Jewitt, D. C. 1992, in Proc. 30th Liège Int. Astrophys. Colloq., ed. A. Brahic, J.-C. Gereard, \& J. Surdej (Liège: Univ. Liège Press), 85

Jewitt, D. 1999, Earth Moon Planets, 79, 35

Lisse, C. M., et al. 2009, PASP, 121, 968

Richardson, J. E., Melosh, H. J., Lisse, C. M., \& Carcich, B. 2007, Icarus, 190, 357

Samarasinha, N. H., A’Hearn, M. F., Hoban, S., \& Klinglesmith, D. A. 1986, in ESA Proc. 20th ESLAB Symp. on the Exploration of Halley's Comet (ESA SP-250, Vol. 1; Noordwijk: ESA), 487

Snodgrass, C., Fitzsimmons, A., Lowry, S. C., \& Weissman, P. 2011, MNRAS, in press 